\title{
DIFERENÇAS ENTRE GÊNERO E PERFIL SÓCIO-ECONÔMICO NO EXAME NACIONAL DE DESEMPENHO DO ESTUDANTE
}

\author{
Marjorie Cristina Rocha da Silva* \\ Claudettte Maria Medeiros Vendramini** \\ FERNANDA LUZIA LOPES***
}

Recebido em: 10 de junho de $2010 \quad$ Aprovado em: 12 de agosto de 2010

\begin{abstract}
*Psicóloga, Mestre e Doutoranda em Psicologia pelo Programa de Pós-Graduação Stricto Sensu da Universidade São Francisco, Itatiba/ SP, Brasil. Membro do grupo de pesquisa do Laboratório de Avaliação Psicológica e Educacional - LabAPE da Universidade São Francisco. Bolsista CAPES/Observatório da Educação. E-mail: silvamarjorie@yahoo.com.br

**Graduada e mestre em Estatística e doutora em Educação, na área de psicologia da Educação Matemática, pela Universidade Estadual de Campinas - UNICAMP. Docente da graduação em Psicologia e pesquisadora do Programa de Pós-Graduação Stricto Sensu em Psicologia da Universidade São Francisco, Itatiba/ SP, Brasil.. Membro dos grupos de pesquisa em Psicologia da Educação Matemática da FE-UNICAMP e do Laboratório de Avaliação Psicológica e Educacional - LabAPE da Universidade São Francisco. Multiplicadora do Programa de Capacitação de Avaliadores de IES do BASis/INEP/MEC. E-mail: cvendramini@uol.com.br ***Psicóloga e mestranda em Psicologia pelo Programa de Pós-Graduação Stricto Sensu da Universidade São Francisco, Itatiba/ SP, Brasil.. Membro do grupo de pesquisa do Laboratório de Avaliação Psicológica e Educacional - LabAPE da Universidade São Francisco. Bolsista CAPES/Observatório da Educação. Email: fernandallopes@hotmail.com
\end{abstract}

Resumo: No contexto atual da educação, o estudo de variáveis relacionadas às diferenças de gênero em estudantes do ensino superior contribui para a compreensão de variáveis associadas ao desempenho acadêmico bem sucedido. Assim, esta pesquisa objetivou verificar em que medida o desempenho dos estudantes no Exame Nacional de Desempenho dos Estudantes - ENADE, do Brasil, variou segundo gênero, e variáveis socioeconômicas. Foram utilizados dados de 147.600 ingressantes e 129.876 concluintes de diversos cursos de graduação que fizeram o ENADE em 2005, selecionados por amostragem aleatória estratificada. Os estudantes responderam a 110 questões sobre o seu perfil socioeconômico e cultural. Os homens obtiveram desempenho médio significativamente maior que as mulheres, nos dois componentes, formação geral e conhecimentos específicos, nos cursos de Matemática, Letras, Biologia, História, Geografia e Filosofia. Observou-se certa tendência de modificação ou até inversão de papéis em áreas reconhecidas tradicionalmente como estereotipadas, o que pode estar refletindo no processo de ensino e relações sociais estabelecidas entre colegas e professores.

Palavras-chave: Avaliação educacional. Ensino superior. Aptidão.

GENDER DIFFERENCES IN THE NATIONAL EXAM OF STUDENTS PERFORMANCE IN BRAZIL

Abstract: The study of variables related to gender differences among undergraduates contributes to the comprehension of variables associated to their successful performance. Therefore, this research aimed to verify how much the students performance at Brazilian Exam of Students Performance - ENADE, varied according to gender, and social-economic variables. The data used referred to 147,600 first-year and 129,876 senior students of several graduation courses who took ENADE exam in 2005 , selected by stratified random sampling. The students answered a questionnaire with 110 questions on their cultural and social-economic profile. Males obtained statistically higher means than females on both the general formation component and the specific knowledge in 
Mathematics, Language, Biology, geography and Philosophy. Observed tendency of modification or even reversal of roles in areas traditionally recognized as stereotyped, which may reflect the process of education and social relations between peers and teachers.

Key words: Educational Evaluation, Higher Education, Ability.

\section{INTRODUÇÃO}

A avaliação educacional, especialmente a partir dos anos 90, passou a ser usada, no contexto brasileiro, como tentativa de encontrar um caminho para a solução de alguns problemas educacionais mais emergentes, a fim de que os processos avaliativos determinassem, entre outros resultados, a elevação dos padrões de desempenho. No entanto, para que isso ocorra é necessária uma reflexão sobre as avaliações operacionalizadas nos vários níveis do sistema educacional, especialmente nas avaliações em larga escala de natureza amostral e supostamente consideradas representativas em termos estatísticos (VIANNA, 2003). Devido ao impacto dessas avaliações nos sistemas educativos, bem como nas políticas públicas, é importante verificar quais fatores estão associados à aquisição de conhecimento e êxito acadêmico, bem como a adequação dos sistemas de avaliação e das interpretações das medidas.

No que se refere à avaliação em larga escala no âmbito educacional brasileiro, existem algumas formas nacionais de avaliação das habilidades e competências estudantis, cabendo destaque ao Sistema de Avaliação do Ensino Básico (SAEB), o Exame Nacional do Ensino Médio (ENEM) e o Exame Nacional de Desempenho do Estudante (ENADE). Esse último se refere à avaliação do conhecimento adquirido ao longo do curso universitário e é o foco do presente estudo.

Autores como Soares (2004) e Ribeiro, Almeida e Gomes (2006) apontam que os fatores que determinam o desempenho acadêmico dizem respeito à estrutura escolar, os associados à família e àqueles relacionados ao próprio estudante. De forma particular, mostraram que tanto o acesso à educação como os resultados escolares estão associados de maneira forte e direta às características socioeconômicas e culturais dos estudantes, além do potencial dos mesmos para aprender. Nessa mesma linha, outros estudos têm apontado que o desempenho acadêmico é mais influenciado pelas características pessoais dos estudantes e das escolas frequentadas. (BRANDÃO, 2000; CÉSAR; SOARES, 2001; FERRÃO; FERNANDES, 2003; FORMIGA, 2004; SOARES, 2004)

Além disso, para que uma avaliação se efetive com êxito, também é importante a elaboração adequada das provas utilizadas e a qualidade científica 
(validade) dessas provas (URBINA, 2004). Alves (1998) ressalta que algumas variáveis têm mostrado uma influência significativa nos resultados dos testes educacionais, o que leva à necessidade de considerá-las e controlá-las em estudos com os mesmos. Entre elas, pode-se destacar o nível sócio-econômico, idade, gênero e grau de escolaridade dos pais.

Rodrigues, Barbosa e Blois (2006) realizaram um estudo de levantamento a respeito das variáveis apontadas nos estudos como relacionadas ao rendimento acadêmico, e verificou-se que as características pessoais, familiares e escolares tiveram um maior destaque como foco destas investigações. Admite-se que variáveis como gênero, idade, atividade laboral, bem como as origens familiares possam influenciar, mas em menor valor, o rendimento acadêmico. Entretanto, o nível de qualidade da escolarização que antecede ao Ensino Superior parece determinar o êxito e o fracasso na maior parte dos estudos encontrados.

Também Carvalho (2000) destaca que, tradicionalmente, tem-se atribuído a responsabilidade à família, pelo sucesso e fracasso escolar dos estudantes. Desta forma, entende-se que o sucesso escolar tem dependido, em grande parte, do apoio direto e sistemático da família que investe nos filhos, compensando tanto dificuldades individuais quanto deficiências escolares. Porém, essa autora ressalta que para isso acontecer, é necessário que a família seja dotada de recursos econômicos e culturais, dentre os quais se destacam o tempo livre para dedicação aos filhos e o nível de escolarização desses pais. Entretanto, por ser considerada como natural essa expressão de dever dos pais, o apoio da família ao sucesso escolar ainda permanece mais implícito do que explícito na pesquisa e política educacional, bem como na prática escolar. Igualmente implícitas permanecem as relações de classe e, sobretudo, de gênero, que compõem os modelos de família, que assim conduzem ao sucesso ou ao fracasso escolar, e que os estudantes carregam ao longo de seu percurso acadêmico e profissional.

No Brasil, a discussão sobre o desempenho bem sucedido nos diferentes níveis de ensino precisa ser precedida do estudo de padrões, para homens e mulheres, de realização educacional, conforme indicam Alves e Beltrão (2004). Também ressaltam que se deve, primeiramente, reconhecer que o resultado que aponta o nível socioeconômico médio da escola como a variável chave na diminuição da desigualdade de desempenho entre os gêneros é bastante plausível. Entende-se desta forma que, famílias em situações econômicas e culturais privilegiadas, freqüentemente estão em condições materiais e ideológicas mais favoráveis para o rompimento de papéis tradicionais em relação a gênero. Finalmente, os autores comentam que grande parte dos pesquisadores 
que investigaram a relação entre gênero e aprendizagem por meio de abordagem qualitativa, insiste que a produção de diferença de gênero envolve viés cultural relacionado com o gênero no desenvolvimento dos currículos das disciplinas e com a própria ação de professores, em sala de aula.

Atualmente, a desigualdade de gênero torna-se evidente em diversos setores de nossa sociedade: no mercado de trabalho, escolha da carreira profissional e remuneração; na política; nas relações sociais, na família; e, na educação. No que se refere à educação, os dados do Censo do Instituto Brasileiro de Geografia e Estatística (IBGE) do ano de 2000 revelam que o nível de escolaridade formal da população brasileira tem se elevado continuamente ao longo dos anos. Em 1976, 35\% dos homens e igual proporção das mulheres não tinham nenhuma instrução ou haviam cursado menos de um ano de escola; em 2002, aproximadamente $12 \%$ tanto dos homens quanto das mulheres brasileiras apresentaram nível de escolaridade tão incipiente. (ANDRADE; FRANCO; PITOMBEIRA, 2003)

Mas no caso das mulheres, outra tendência de especial importância para a sua inserção no mercado de trabalho, se esboçou e concretizou nos últimos 25 anos. Comparativamente aos homens, as mulheres brasileiras adquiriram maior nível de escolaridade. No ensino superior, bem como na educação profissional, quando se observam as opções femininas segundo as áreas de conhecimento, nota-se a existência de algumas mais permeáveis à presença das mulheres e outras nem tanto, como ressalta Barroso (2004). Em contrapartida, em 2002 elas representam 75\% dos que optaram por Educação, 63\% dentre os que escolheram cursos na área de Humanidades e Artes e 72\% dos que pensavam em atuar no futuro em carreiras da Saúde e do Bem-estar Social.

Nessa mesma linha de investigação, um estudo realizado em Portugal por Almeida e outros (2006) analisou o impacto do gênero e da origem sócio-cultural na nota de vestibular e nos cursos escolhidos no Ensino Superior, assim como nas dificuldades antecipadas e no desempenho acadêmico ao final do primeiro ano universitário. O estudo investigou 1.407 estudantes de vários cursos da Universidade do Minho. Os resultados apontam que o gênero e o nível sóciocultural das famílias influenciam a escolha de cursos, mais estudantes do sexo feminino e das classes mais desfavorecidas freqüentam cursos de Ciências Sociais, ao passo que uma maior parcela de estudantes do sexo masculino e das classes mais favorecidas o fazem em cursos de Engenharia. Verifica-se ainda que os estudantes do sexo feminino e mais favorecidos socialmente apresentam notas mais elevadas tanto no vestibular quanto no desempenho médio do primeiro ano. 
Partindo do pressuposto de que nem a categoria mulher nem a categoria homem são homogêneas, Saavedra (2001) realizou um estudo em que estas categorias foram estudadas em relação ao nível socioeconômico, em que analisou, portanto se existia uma relação entre as classificações escolares, o sexo e o nível socioeconômico dos estudantes. Participaram do estudo 591 estudantes do $7^{\circ}$ e $9^{\circ}$ ano de três escolas de Portugal, sendo $53,3 \%$ do gênero masculino. Foi possível concluir que a escola continua a ser um espaço de desigualdade social, tanto no que diz respeito ao gênero quanto ao nível socioeconômico. No que diz respeito ao gênero, as desigualdades são, contudo, de menores dimensões se comparadas à classe social. As mulheres, mesmo das classes mais baixas, parecem tender a igualar-se aos seus pares e, até por vezes superá-los em disciplinas consideradas tipicamente masculinas. Verifica-se, portanto, que as mulheres conseguem ultrapassar melhor que os homens as desvantagens do seu nível socioeconômico. Nota-se que os estereótipos de gênero continuam presentes, embora reduzidos neste estudo a duas disciplinas: o Português para o feminino e a Educação Física para o masculino.

Segundo a referida autora, a problemática dos estereótipos nas disciplinas e cursos acadêmicos prende-se à chamada bipolarização dos conhecimentos, ou seja, que os homens escolhem e são melhores alunos no campo das Ciências (sobretudo em Física), Matemática e Tecnologias (como Arquitetura e Engenharias), e as mulheres no campo das Línguas e Humanas. Ainda, segundo a mesmo autora, esta bipolarização de conhecimentos reflete o dualismo do gênero, que está profundamente enraizado na linguagem e na cultura. Assim, os atributos geralmente associados ao gênero feminino, como intuição, emocionalidade, subjetividade, expressividade e sensibilidade, estariam associados às Línguas, Humanas e Artes. E, os supostos traços masculinos, de racionalidade, objetividade, impassibilidade e impessoalidade, encontram-se associados às Ciências Físicas, Tecnológicas e à Matemática.

Autores como Andrade, Franco e Pitombeira (2003) destacam que alguns aspectos importantes na relação entre gênero e desempenho, em uma determinada disciplina ou curso, devem ser especialmente incluídos e investigados, tais como: variáveis cognitivas (níveis e tipos de raciocínio envolvidos, insegurança, persistência, dedicação), certos aspectos sociais (nível socioeconômico, raça); influência dos pais; atuação do professor no ambiente da sala de aula; entre outros. Esse autor conclui que as preferências e dificuldades relacionadas a disciplinas específicas, repercutem na escolha de cursos no ensino superior.

Portanto, o presente estudo teve por objetivo verificar em que medida o desempenho dos estudantes no ENADE variou segundo o gênero dos mesmos 
e interagiu com outras variáveis apontadas como relevantes em alguns dos estudos anteriormente apresentados, a saber, etnia e faixa de renda mensal da família. Desta maneira, considera-se relevante investigar o quanto algumas das variáveis do perfil socioeconômico do estudante podem estar relacionadas ao desempenho na prova, e como essas diferenças ocorrem entre os gêneros.

\section{MÉTODO}

\section{Fonte de dados}

Foi utilizado o Banco de dados do Exame Nacional do Desempenho do Estudante (ENADE) contendo informações acadêmicas e os resultados dos estudantes submetidos ao ENADE em 2005. Participaram neste ano 277.476 estudantes (147.600 ingressantes e 129.876 concluintes), selecionados pelo INEP por procedimentos de amostragem aleatória estratificada, provenientes das seguintes áreas: Arquitetura e Urbanismo, Biologia, Ciências Sociais, Computação, Engenharia (oito grupos), Filosofia, Física, Geografia, História, Letras, Matemática, Pedagogia e Química. Do total de participantes, 56,9\% são do gênero feminino. A Tabela 1 apresenta a distribuição dos estudantes por cursos participantes.

Observa-se que a carreira com maior número de participantes é Pedagogia, seguida das Engenharias e Letras, que totalizam 52,1\% do total de estudantes. As carreiras de Ciências Sociais, Física e Filosofia apresentaram o menor número de participantes. De acordo com o Resumo Técnico de 2005, houve um crescimento de 152,3\% no número de participantes em relação a 2004, o que pôde ser verificado em todas as regiões do país.

O ENADE é uma prova de 40 questões avaliando conhecimentos, competências profissionais e habilidades acadêmicas. Dessa prova, 10 questões correspondem ao componente de Formação Geral (FG), avaliando aspectos mais gerais da formação e 30 questões específicas de cada área (CE). A cada questão correta é atribuído um ponto e a cada questão discursiva uma proporção de 0 a 1 . Ao final tem-se uma nota, para cada estudante, em uma escala de 0 a 100 correspondendo à porcentagem de pontos corretos conseguidos.

\section{QUESTIONÁRIO SOCIOECONÔMICO}

Além do ENADE, os estudantes respondem a um questionário socioeconômico com 110 questões, indagando sobre perfil socioeconômico e cultural, além de aspectos relacionados à dedicação em atividades acadêmicas, e percepção de 
DIFERENÇAS ENTRE GÊNERO E PERFIL SÓCIO-ECONÔMICO NO EXAME NACIONAL DE DESEMPENHO DO ESTUDANTE

Tabela 1. Distribuição dos estudantes e cursos submetidos ao ENADE em 2005

\begin{tabular}{l|r|r|r|r}
\hline Cursos & Ingressantes & Concluintes & \multicolumn{1}{|c|}{ Total } & \multicolumn{1}{c}{$\%$} \\
\hline Arquitetura e Urbanismo & 3.621 & 2.912 & 6.533 & 2,4 \\
\hline Biologia & 13.542 & 10.933 & 24.475 & 8,8 \\
\hline Ciências Sociais & 1.680 & 2.150 & 3.830 & 1,4 \\
\hline Computação & 18.004 & 14.056 & 32.060 & 11,6 \\
\hline Engenharia-Grupo I & 4.562 & 4.163 & 8.725 & 3,1 \\
\hline Engenharia-Grupo II & 10.154 & 7.012 & 17.166 & 6,2 \\
\hline Engenharia-Grupo III & 2.598 & 1.878 & 4.476 & 1,6 \\
\hline Engenharia-Grupo IV & 2.954 & 2.185 & 5.139 & 1,9 \\
\hline Engenharia-Grupo V & 712 & 449 & 1.161 & 0,4 \\
\hline Engenharia-Grupo VI & 4.751 & 2.164 & 6.915 & 2,5 \\
\hline Engenharia-Grupo VII & 2.639 & 779 & 3.418 & 1,2 \\
\hline Engenharia-Grupo VIII & 1.412 & 923 & 2.335 & 0,8 \\
\hline Filosofia & 2.363 & 2.056 & 4.419 & 1,6 \\
\hline Física & 2.574 & 1.654 & 4.228 & 1,5 \\
\hline Geografia & 5.668 & 6.075 & 11.743 & 4,2 \\
\hline História & 9.537 & 9.075 & 18.162 & 6,5 \\
\hline Letras & 23.330 & 22.870 & 46.200 & 16,7 \\
\hline Matemática & 10.229 & 9.243 & 19.472 & 7,0 \\
\hline Pedagogia & 23.318 & 26.179 & 49.497 & 17,8 \\
\hline Química & 3.952 & 3.120 & 7.072 & 2,5 \\
\hline Total & 147.600 & 129.876 & 277.476 & 100,0 \\
\hline Fia & & &
\end{tabular}

Fonte: Relatório Técnico - ENADE/2005. Disponível em: <www.inep.gov.br/enade>.

elementos da instituição e da formação por ela oferecida. Nesse questionário, é possível, extrair várias dimensões importantes a respeito do perfil dos estudantes e, também, via agregação, é possível obter dados contextuais característicos dos diferentes cursos.

Algumas inferências importantes foram ressaltadas no Relatório Técnico de 2005 e cabe aqui serem retomadas. Entre os ingressantes das vinte áreas examinadas, as que concentram o maior número de ingressantes e concluintes provenientes de famílias de baixa renda são especialmente as licenciaturas (Geografia, Pedagogia, Letras, História, Física, Química, Matemática, Filosofia e Biologia). Cresceu significativamente o percentual (de 20,7\% em 2002 para $45,4 \%$ em 2005) de estudantes que se declaram filhos de pais sem escolaridade ou com escolaridade até a $4^{\text {a }}$ série do ensino fundamental. Tem havido um 
constante crescimento percentual de alunos originários da escola média pública (de 25\% em 2002 para 58,8\% em 2005).

\section{PROCEDIMENTO}

Após a autorização para a utilização do banco de dados do ENADE 2005, realizou-se a presente pesquisa que manteve o sigilo dos dados individuais e também das IES. A análise dos dados foi realizada com o auxílio do programa estatístico SPSS for Windows (2002).

\section{RESULTADOS E DISCUSSÃO}

A fim de verificar as possíveis variabilidades de desempenho segundo o gênero dos participantes e devido à diversidade de áreas no ENADE 2005, os estudantes foram analisados separadamente por curso e desempenho na prova de Formação Geral e de Componente Específico, como pode ser visualizado na Tabela 2.

De acordo com as análises de $t$ de Student para analisar diferenças de média entre os gêneros, somente em nove carreiras não foram verificadas médias estatisticamente diferentes a favor de um dos gêneros, em pelo menos uma das partes da prova. No que se refere à prova de Formação de Geral, não foram encontradas diferenças estatisticamente significativas de desempenho por gênero nos cursos de Física $(t[2424,5]=-1,895 ; p=0,058)$; Química $(t[6763,3]=-1,001 ; p=0,317)$; Computação $(t[9867,7]=-1,818 ; p=0,069)$; Engenharia-Grupo V $(t[1158]=-$ $1,558 ; p=0,120)$ e Engenharia-Grupo VIII $(t[2333]=-0,432 ; p=0,666)$. Em relação ao Componente Específico, não foram encontradas diferenças estatisticamente significativas entre os gêneros nos cursos de Química $(t[6713,4]=1,176$; $p=0,240)$; Arquitetura e Urbanismo $(t[6531]=0,070 ; p=0,944)$; EngenhariaGrupo III $(t[4474]=1,146 ; p=0,252)$; Engenharia-Grupo IV $(t[4607,5]=0,170$; $p=0,865)$ e Engenharia-Grupo VI $(t[6911]=-0,392 ; p=0,695)$.

Na parte de Formação Geral, os estudantes do gênero masculino obtiveram médias estatisticamente maiores que do feminino nos cursos de Matemática, Letras, Biologia, Pedagogia, História, Geografia e Filosofia. Nesta mesma parte da prova, as mulheres obtiveram médias estatisticamente maiores que dos homens nos cursos de Arquitetura e Urbanismo, Ciências Sociais e Engenharias dos Grupos I, II, III, IV, VI e VII. Essas informações podem também ser bem visualizadas na Figura 1, que mostra a média (traço ao centro) e o erro-padrão da média (linha), por gênero, em cada um dos cursos. 
DIFERENÇAS ENTRE GÊNERO E PERFIL SÓCIO-ECONÔMICO NO EXAME NACIONAL DE DESEMPENHO DO ESTUDANTE

Tabela 2. Desempenho dos estudantes segundo curso e gênero

\begin{tabular}{|c|c|c|c|c|c|c|}
\hline \multirow[b]{2}{*}{ Carreira } & \multirow[b]{2}{*}{$\begin{array}{l}\text { Número de } \\
\text { estudantes }\end{array}$} & \multirow[b]{2}{*}{$\begin{array}{l}\text { Nota nas duas } \\
\text { partes da prova }\end{array}$} & \multicolumn{2}{|c|}{ Masculino } & \multicolumn{2}{|c|}{ Feminino } \\
\hline & & & $\begin{array}{c}\% \text { de } \\
\text { estudantes }\end{array}$ & $\begin{array}{c}\text { Nota } \\
\text { Média }\end{array}$ & $\begin{array}{c}\% \text { de } \\
\text { estudantes }\end{array}$ & $\begin{array}{l}\text { Nota } \\
\text { Média }\end{array}$ \\
\hline \multirow[t]{2}{*}{ Matemática } & \multirow[t]{2}{*}{19.466} & Formação Geral & $46 \%$ & 54,6 & $54 \%$ & 53,6 \\
\hline & & C. Especifico & & 25,0 & & 23,9 \\
\hline \multirow[t]{2}{*}{ Letras } & \multirow[t]{2}{*}{46.200} & Formação Geral & $18 \%$ & 58,4 & $82 \%$ & 56,4 \\
\hline & & C. Especifico & & 29,0 & & 28,3 \\
\hline \multirow[t]{2}{*}{ "Fisica } & \multirow[t]{2}{*}{4.228} & Formação Geral & $73 \%$ & 53,7 & $27 \%$ & 55,2 \\
\hline & & C. Especifico & & 26,3 & & 24,4 \\
\hline \multirow[t]{2}{*}{ Quimica } & \multirow[t]{2}{*}{7.072} & Formação Geral & $47 \%$ & 52,5 & $53 \%$ & 52,9 \\
\hline & & C. Especifico & & 25,1 & & 24,7 \\
\hline \multirow[t]{2}{*}{ Biologia } & \multirow[t]{2}{*}{24.474} & Formação Geral & $29 \%$ & 58,5 & $71 \%$ & 58,0 \\
\hline & & C. Especifico & & 24,3 & & 23,4 \\
\hline \multirow[t]{2}{*}{ Pedagogia } & \multirow[t]{2}{*}{49.497} & Fommação Geral & $8 \%$ & 50,2 & $92 \%$ & 49,0 \\
\hline & & C. Especifico & & 39,5 & & 41,7 \\
\hline \multirow{2}{*}{$\begin{array}{l}\text { Arquitetura e } \\
\text { Urbanismo }\end{array}$} & \multirow[t]{2}{*}{6.533} & Formação Geral & $35 \%$ & 48,9 & $65 \%$ & 50,4 \\
\hline & & C. Especifico & & 39,3 & & 39,3 \\
\hline \multirow[t]{2}{*}{ Históna } & \multirow[t]{2}{*}{18.612} & Formação Geral & $43 \%$ & 54,5 & $57 \%$ & 52,2 \\
\hline & & C. Especifico & & 359 & & 30,9 \\
\hline \multirow[t]{2}{*}{ Geografia } & \multirow[t]{2}{*}{11.743} & Formação Geral & $46 \%$ & 60,0 & $54 \%$ & 58,5 \\
\hline & & C. Especifico & & 32,6 & & 30,7 \\
\hline \multirow[t]{2}{*}{ Filosofia } & \multirow[t]{2}{*}{4.413} & Fommação Geral & $67 \%$ & 55,8 & $33 \%$ & 53,7 \\
\hline & & C. Especifico & & 26,1 & & 24,2 \\
\hline Computação & 32.039 & Formação Geral & $81 \%$ & 55,0 & $19 \%$ & 55,5 \\
\hline & & C. Especifico & & 27,5 & & 25,8 \\
\hline Ciências Sociais & 3.829 & Formação Geral & $44 \%$ & 45,1 & $56 \%$ & 48,5 \\
\hline & & C. Especifico & & 39,6 & & 41,5 \\
\hline Engenharia - & 8.725 & Formação Geral & $78 \%$ & 54,2 & $22 \%$ & 55,6 \\
\hline Grupo I & & C. Especifico & & 33,4 & & 32,6 \\
\hline Engenharia - & 17.166 & Formação Geral & $91 \%$ & 54,1 & $9 \%$ & 56,3 \\
\hline Grupo II & & C. Especifico & & 29,0 & & 27,3 \\
\hline Engenharia - & 4.476 & Formação Geral & $94 \%$ & 56,5 & $6 \%$ & 58,8 \\
\hline Grupo III & & C. Especifico & & 36,4 & & 35,3 \\
\hline Engenharia - & 5.137 & Formação Geral & $44 \%$ & 59,0 & $56 \%$ & 61,2 \\
\hline Grupo IV & & C. Especifico & & 35,3 & & 35,3 \\
\hline Engenharia - & 1.160 & Formação Geral & $77 \%$ & 62,8 & $23 \%$ & 64,5 \\
\hline Grupo V & & C. Especifico & & 41,0 & & 37,3 \\
\hline Engenharia - & 6.914 & Formação Geral & $79 \%$ & 59,2 & $21 \%$ & 61,7 \\
\hline Grupo XI & & C. Especifico & & 38,4 & & 38,6 \\
\hline Engenharia - & 3.417 & Formação Geral & $67 \%$ & 58,7 & $33 \%$ & 61,7 \\
\hline Grupo VII & & C. Especifico & & 37,8 & & 36,2 \\
\hline Engenharia - & 2.335 & Formação Geral & $67 \%$ & 49,1 & $33 \%$ & 49,5 \\
\hline Grupo VIII & & C. Especifico & & 31,0 & & 27,4 \\
\hline
\end{tabular}

No Componente Específico, os estudantes do gênero masculino obtiveram médias estatisticamente maiores que do feminino nos cursos de Matemática, Letras, Física, Biologia, História, Geografia, Filosofia, Computação e Engenharias I, II,V, VII e VIII. Nesta mesma parte da prova, as mulheres obtiveram médias estatisticamente maiores que dos homens somente nos cursos de Pedagogia e Ciências Sociais. Essas informações podem também ser bem visualizadas na Figura 2. 


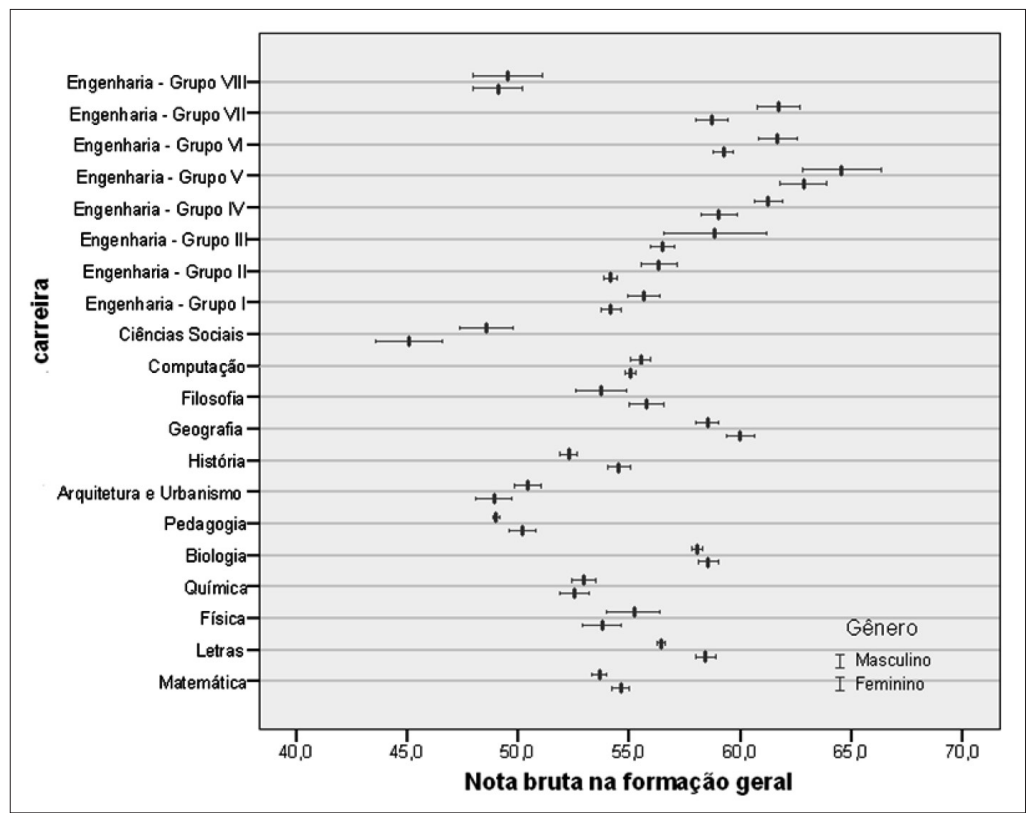

Figura 1. Intervalos de confiança das notas na Formação Geral, por curso e gênero

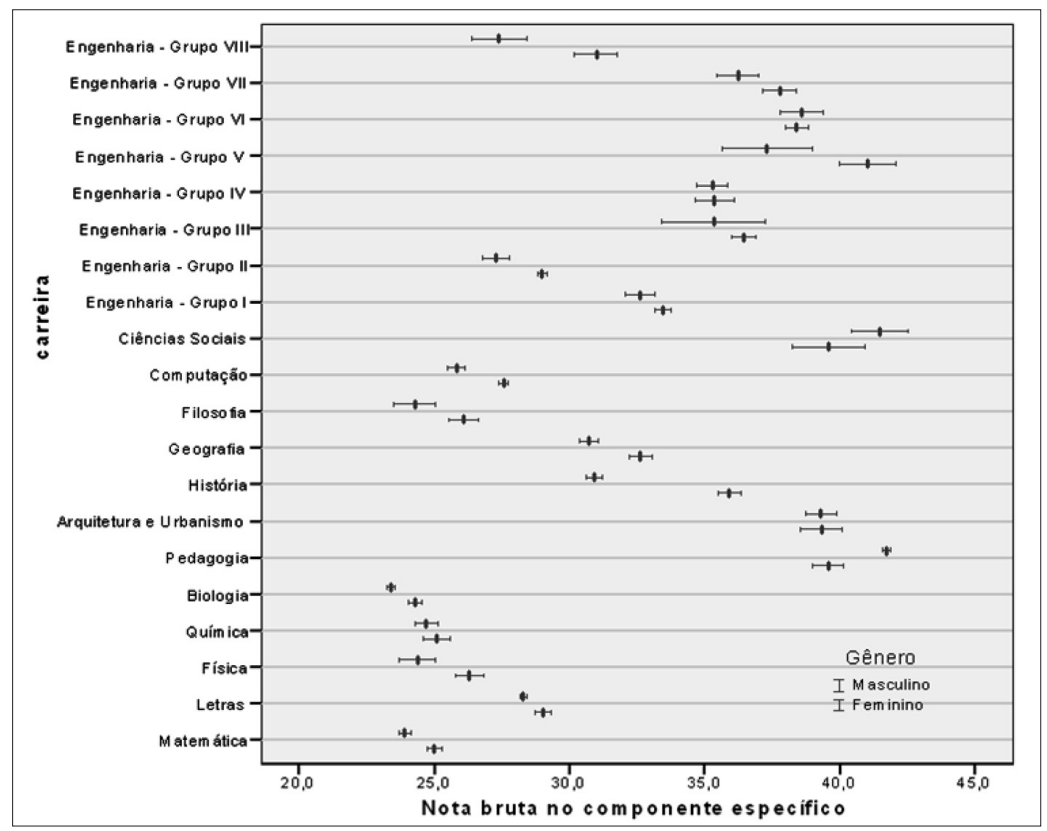

Figura 2. Intervalos de confiança das notas no Componente Específico, por curso e gênero 
Esses resultados vão ao encontro de pesquisas realizadas por Almeida e outros (2006), Barroso (2004) e Saavedra (2001), que apontam que em algumas áreas de exatas, como a Matemática e Engenharia, ainda há a tendência dos homens obterem maiores médias. Porém, esses autores demonstram, como também apontado por Saavedra (2001), que existe uma tendência à modificação e/ou inversão destes estereótipos, visto que também na presente pesquisa foram observados resultados bastante diversificados da tendência tradicional. Isso pode ser observado, por exemplo, em cursos como Letras, Biologia, Filosofia entre outras, em que houve uma diferença a favor dos homens. Outro destaque deve ser dado aos resultados na parte de Formação Geral, em que as diferenças entre os gêneros mostram-se bem diferentes do que normalmente apontado, tendo, por exemplo, maiores médias para mulheres em áreas mais masculinas, como Engenharia e Arquitetura. Portanto, destacam-se as reflexões de autores como Andrade, Franco e Pitombeira (2003) sobre a necessidade de se investigar outras variáveis importantes relacionadas ao desempenho, como aspectos cognitivos e culturais dos estudantes.

A fim de verificar se algumas outras variáveis apontadas na revisão teórica como passíveis de influência no desempenho acadêmico também se comportam da mesma forma no presente estudo, procedeu-se à análise de diferença de média ANOVA também por tipo de Etnia, faixa de renda mensal da família e grau de escolaridade dos pais. No que tange à Etnia dos participantes, verifica-se pela Tabela 3 que a maior parte dos estudantes são brancos $(65,1 \%)$.

Tabela 3. Distribuição dos estudantes segundo etnia e gênero

\begin{tabular}{l|r|r|r}
\hline \multirow{2}{*}{ Etnia } & \multicolumn{2}{|c|}{ Gênero } & \multirow{2}{*}{$\%$ Total } \\
\cline { 2 - 3 } & Masculino & \multicolumn{1}{|c}{ Feminino } & \\
\hline Branco/a & 57.268 & 80.788 & 65,1 \\
\hline Negro/a & 5.388 & 7.747 & 6,2 \\
\hline Pardo/a, Mulato/a & 21.059 & 33.634 & 25,8 \\
\hline Amarelo/a (de origem oriental) & 1.749 & 2.104 & 1,8 \\
\hline Indígena ou de origem indígena & 993 & 1.372 & 1,1 \\
\hline Total & 86.457 & 125.645 & 100,0 \\
\hline
\end{tabular}

Ao cruzar essas informações segundo os cursos participantes, verifica-se que mais de $60,0 \%$ dos estudantes em cada curso são brancos. Ao analisar o desempenho dos estudantes nas duas partes da prova pela Etnia, verificou-se uma diferença de média estatisticamente significativa na grande maioria dos cursos (Tabela 4). Em relação à Formação Geral, não foram verificadas dife- 
renças significativas entre os cursos de Engenharia do Grupo IV, VII e VIII. No que se refere ao Componente Específico, não foram constatadas diferenças estatisticamente significativas entre os grupos nos cursos de Ciências Sociais e na Engenharia do grupo VII.

Tabela 4. Análise de variância para testar diferenças de desempenho dos estudantes por etnia

\begin{tabular}{|c|c|c|c|c|c|c|}
\hline Cursos & $\begin{array}{c}\text { Nota nas partes } \\
\text { da prova }\end{array}$ & $\begin{array}{c}\text { Soma de } \\
\text { quadrados }\end{array}$ & $\begin{array}{l}\text { Graus de } \\
\text { liberdade }\end{array}$ & $\begin{array}{c}\text { Quadrado } \\
\text { Médio }\end{array}$ & $\begin{array}{c}\text { Estatistica } \\
F \\
\end{array}$ & $\begin{array}{c}\text { Significância do } \\
\text { teste }(p)\end{array}$ \\
\hline \multirow[t]{2}{*}{ Matemática } & FG & 4115215 & 4 & 1028,804 & 3,389 & 0,009 \\
\hline & $\mathrm{CE}$ & 6474.608 & 4 & 1618,652 & 10,838 & $Q, 000$ \\
\hline \multirow[t]{2}{*}{ Letras } & FG & 41385,854 & 4 & 10346,463 & 31,160 & $Q, 000$ \\
\hline & $\mathrm{CE}$ & 27317,878 & 4 & 6829470 & 40,890 & Q,000 \\
\hline \multirow[t]{2}{*}{ Fisica } & FG & 16135535 & 4 & 4033884 & 7,471 & 2,000 \\
\hline & $\mathrm{CE}$ & 9801,662 & 4 & 2450416 & 13,072 & 0,000 \\
\hline \multirow[t]{2}{*}{ Química } & FG & 3933566 & 4 & 283,392 & 2989 & 0,018 \\
\hline & $\mathrm{CE}$ & 4292,202 & 4 & 1073.051 & 6.223 & 0,000 \\
\hline \multirow[t]{2}{*}{ Biologa } & $\mathrm{FG}$ & 18340,086 & 4 & 4585021 & 14,285 & 2,000 \\
\hline & $\mathrm{CE}$ & 5129596 & 4 & 1282,399 & 13,109 & 0,000 \\
\hline \multirow[t]{2}{*}{ Pedagoga } & $F G$ & 13543,015 & 4 & 3385.754 & 12,274 & 0,000 \\
\hline & $C E$ & 37491,041 & 4 & 2372,760 & 31,324 & 2,000 \\
\hline \multirow{2}{*}{$\begin{array}{l}\text { Arquitetura e } \\
\text { Urbanismo }\end{array}$} & FG & 6253960 & 4. & 1563,490 & 3,881 & 0,004 \\
\hline & $\mathrm{CE}$ & 10735,362 & 4 & 2683841 & 7,550 & 0,000 \\
\hline \multirow[t]{2}{*}{ Históna } & FG & 27445937 & 4 & 6861484 & 14,590 & 2,000 \\
\hline & $\mathrm{CE}$ & 18307,329 & 4 & 4576832 & 15,649 & 0,000 \\
\hline \multirow[t]{2}{*}{ Geografia } & FG & 21856,376 & 4 & 5464094 & 11754 & 2,000 \\
\hline & $C E$ & 15043,429 & 4 & 3760857 & 17,685 & 0,000 \\
\hline \multirow[t]{2}{*}{ Filosofia } & FG & 7334.851 & 4 & 1833.713 & 4,691 & 0,001 \\
\hline & $\mathrm{CE}$ & 8157154 & 4 & 2039289 & 2162 & 0,000 \\
\hline \multirow[t]{2}{*}{ Computação } & FG & 3697976 & 4 & 224.494 & 2,779 & $Q, 025$ \\
\hline & $\mathrm{CE}$ & 10681,738 & 4 & 2670,434 & 13.597 & 0,000 \\
\hline \multirow[t]{2}{*}{ Ciencias Sociais } & FG & 7714,106 & 4 & 1928,527 & 2,360 & 2,051 \\
\hline & $C E$ & 3347301 & 4 & 836.825 & 1,302 & 0,267 \\
\hline \multirow{2}{*}{$\begin{array}{l}\text { Engenharia } \\
\text { Grupo I }\end{array}$} & FG & 6040,109 & 4 & 1510,027 & 4,983 & 0,001 \\
\hline & $\mathrm{CE}$ & $\$ 654976$ & 4 & 2163,744 & 13.474 & 0,000 \\
\hline \multirow{2}{*}{$\begin{array}{l}\text { Engenharia } \\
\text { Grupo II }\end{array}$} & FG & 6674,292 & 4 & 1668,573 & 5,378 & 2,000 \\
\hline & $\mathrm{CE}$ & 9550467 & 4 & 2387617 & 16.678 & 0,000 \\
\hline \multirow{2}{*}{$\begin{array}{l}\text { Engenharia } \\
\text { Grupo III }\end{array}$} & FG & 3908,596 & 4 & 977,149 & 3,351 & 2,010 \\
\hline & $\mathrm{CE}$ & 4343870 & 4 & 1085968 & 4,828 & 0,001 \\
\hline \multirow{2}{*}{$\begin{array}{l}\text { Engenharia } \\
\text { Grupo IV }\end{array}$} & FG & 2634,132 & 4 & 658,533 & 1965 & 0,097 \\
\hline & $\mathrm{CE}$ & 6057007 & 4 & 1514252 & 5546 & 0,000 \\
\hline \multirow{2}{*}{$\begin{array}{l}\text { Engenharia } \\
\text { Grupo V }\end{array}$} & FG & 748.762 & 4 & 187.190 & 264 & 0,549 \\
\hline & $\mathrm{CE}$ & 2247003 & 4 & 561.751 & 2,385 & 0,050 \\
\hline \multirow{2}{*}{$\begin{array}{l}\text { Engenhana. } \\
\text { Grupe XI }\end{array}$} & FG & 2491963 & 4 & 622991 & 2,278 & 2,059 \\
\hline & $\mathrm{CE}$ & 8842961 & 4 & $2210^{740}$ & 2,443 & 0,000 \\
\hline \multirow{2}{*}{$\begin{array}{l}\text { Engenharia } \\
\text { Grupo VII }\end{array}$} & FG & 1292619 & 4 & 323155 & 1,067 & Q.371 \\
\hline & $\mathrm{CE}$ & 1324,714 & 4 & 331,178 & 1,710 & 0,145 \\
\hline \multirow{2}{*}{$\begin{array}{l}\text { Engenharia } \\
\text { Grupo VIII }\end{array}$} & FG & 3020272 & 4 & 755,068 & 1,569 & 0,180 \\
\hline & $\mathrm{CE}$ & 6310,812 & 4 & 1577,703 & 6.807 & 2,000 \\
\hline
\end{tabular}


Dos cursos em que foram verificadas diferenças estatisticamente significativas, examinou-se desempenho ligeiramente maior a favor dos brancos na Formação Geral, exceto nos cursos de Arquitetura e Urbanismo, Ciências Sociais, Filosofia, Computação e Engenharias dos grupos II, III, V e VI. No Componente Específico, da igual forma a favor dos brancos, com exceção dos cursos de Biologia, Computação, e Engenharias dos grupos IV a VI.

Em relação à faixa de renda mensal da família do estudante, verifica-se pela Tabela 5, que a maior parte dos estudantes conta com uma renda de até 10 salários mínimos $(82,1 \%)$.

Tabela 5. Distribuição dos estudantes segundo faixa de renda mensal e gênero

\begin{tabular}{l|r|r|r}
\hline \multirow{2}{*}{ Faixa de renda mensal } & \multicolumn{2}{|c|}{ Gênero } & \multirow{2}{*}{$\%$ Total } \\
\cline { 2 - 3 } & Masculino & Feminino & \\
\hline Até 3 salários-mínimos & 17.508 & 46.280 & 30,2 \\
\hline De 3 a 10 salários-mínimos & 46.091 & 63.606 & 51,9 \\
\hline De 10 a 20 salários-mínimos & 15.401 & 10.786 & 12,4 \\
\hline De 20 a 30 salários-mínimos & 4.320 & 2.792 & 3,4 \\
\hline Mais de 30 salários-mínimos & 2.857 & 1.603 & 2,1 \\
\hline Total & 86.177 & 125.067 & 100,0 \\
\hline
\end{tabular}

Ao analisar o desempenho dos estudantes nas duas partes da prova segundo faixa de renda mensal, verificou-se uma diferença de média estatisticamente significativa na grande maioria dos cursos (Tabela 6). Ao avaliar o desempenho dos estudantes nas duas partes da prova pela renda mensal, verificou-se uma diferença de média estatisticamente maior daqueles estudantes cujas famílias recebem de 10 a 30 salários mínimos em relação aos demais grupos, exceto em alguns cursos. Em relação à Formação Geral, não foram verificadas diferenças entre os grupos nos cursos de Arquitetura e Urbanismo, Ciências Sociais Engenharias VIII. No que se refere ao Componente Específico, não foram constatadas diferenças estatisticamente significativas entre os grupos no curso de Ciências Sociais.

A fim de verificar a influência, conjuntamente, das variáveis gênero, etnia e faixa de renda mensal da família, optou-se pela junção de categorias com uma pequena porcentagem de sujeitos, e que não haviam apresentado diferenças significativas em relação às demais. Desta forma, para a análise multivariada, foram consideradas para a Etnia as categorias Branco, Negro, Pardo e Mulato. $\mathrm{Na}$ faixa de renda salarial mensal, analisou-se as categorias "até 3 salários mínimos", "de 3 a 10 salários" e "acima de 10 salários". Além disso, essas variáveis foram verificadas controlando-se o efeito de cada curso separadamente, já que as 
Tabela 6. Análise de variância para testar diferenças de desempenho dos estudantes por renda

\begin{tabular}{|c|c|c|c|c|c|c|}
\hline Cursos & \begin{tabular}{|c|} 
Nota nas partes da \\
prova
\end{tabular} & $\begin{array}{c}\text { Soma de } \\
\text { quadrados }\end{array}$ & $\begin{array}{l}\text { Graus de } \\
\text { liberdade }\end{array}$ & $\begin{array}{c}\text { Quadrado } \\
\text { Médio }\end{array}$ & $\begin{array}{c}\text { Estatistica } \\
F\end{array}$ & $\begin{array}{l}\text { Significância } \\
\text { do teste }(p)\end{array}$ \\
\hline \multirow[t]{2}{*}{ Matemática } & FG & 23128,596 & 4. & 5782,149 & 19,189 & 0,000 \\
\hline & $\mathrm{CE}$ & 33762,851 & 4 & 8440713 & 57,531 & 0,000 \\
\hline \multirow[t]{2}{*}{ Letras } & FG & 175236,510 & 4 & 43809,127 & 133619 & 0,000 \\
\hline & $\mathrm{CE}$ & 156256,039 & 4 & 39064,010 & 238472 & 0000 \\
\hline \multirow[t]{2}{*}{ Fisica } & FG & 7464,117 & 4 & 1866,029 & 3.445 & 0,008 \\
\hline & $\mathrm{CE}$ & 9422.031 & 4 & 2355508 & 12,554 & 0,000 \\
\hline \multirow[t]{2}{*}{ Quimica } & FG & 63320195 & 4 & 1583,049 & 4,807 & 9,001 \\
\hline & $\mathrm{CE}$ & 7381917 & 4 & 1845479 & 10,696 & 0,000 \\
\hline \multirow[t]{2}{*}{ Biologia } & FG & 94845,529 & 4 & 23711,382 & 74,844 & 0,000 \\
\hline & $\mathrm{CE}$ & 23632,342 & 4 & 5908,086 & 60,967 & 0000 \\
\hline \multirow[t]{2}{*}{ Pedagogia } & FG & 64087,874 & 4 & 16021,968 & 58,406 & 0,000 \\
\hline & $\mathrm{CE}$ & 167603,363 & 4 & 41900.841 & 141.576 & 0,000 \\
\hline \multirow{2}{*}{$\begin{array}{l}\text { Arquitetura e } \\
\text { Urbanismo }\end{array}$} & FG & 3289.444 & 4 & 822,361 & 2,039 & 0,086 \\
\hline & $\mathrm{CE}$ & 13568,149 & 4 & 3322037 & 2552 & 0,000 \\
\hline \multirow[t]{2}{*}{ "Históna" } & FG & 38486,792 & 4 & 2621,698 & 20,381 & 2,000 \\
\hline & $\mathrm{CE}$ & 91550.233 & 4 & 22887,558 & 792289 & 0,000 \\
\hline \multirow[t]{2}{*}{ Geografia } & FG & 28310.792 & 4 & 7077698 & 15,252 & 0,000 \\
\hline & $\mathrm{CE}$ & 50216,610 & 4 & 12554.152 & 59,949 & 0,000 \\
\hline \multirow[t]{2}{*}{ Filosofia } & FG & 6085982 & 4 & 1521,496 & 3,855 & 2,004 \\
\hline & $\mathrm{CE}$ & 13598.497 & 4 & 3399624 & 15.449 & 0,000 \\
\hline \multirow[t]{2}{*}{ Computação } & FG & 55942,988 & 4 & 13985.747 & 42411 & 2,000 \\
\hline & $\mathrm{CE}$ & 100934,151 & 4 & 25233.538 & 131,862 & 0,000 \\
\hline \multirow{2}{*}{$\begin{array}{l}\text { Ciências } \\
\text { Sociais }\end{array}$} & FG & 256.681 & 4 & 64,170 & 078 & 0,989 \\
\hline & $\mathrm{CE}$ & 3725089 & 4 & 231.272 & 1.449 & 0,215 \\
\hline \multirow{2}{*}{$\begin{array}{l}\text { Engenhana } \\
\text { Grupo I }\end{array}$} & FG & 18483,821 & 4 & 4620955 & 15,305 & 0,000 \\
\hline & $\mathrm{CE}$ & 24772,629 & 4 & 6193157 & 39,027 & 0,000 \\
\hline \multirow{2}{*}{$\begin{array}{l}\text { Engenhana } \\
\text { Grupo II }\end{array}$} & FG & 60320,645 & 4 & 15080,161 & 49,118 & 2,000 \\
\hline & $\mathrm{CE}$ & 46413,910 & 4 & 11603,477 & $\$ 2,533$ & 0,000 \\
\hline \multirow{2}{*}{$\begin{array}{l}\text { Engenharia } \\
\text { Grupo III }\end{array}$} & FG & 14020890 & 4 & 3505,222 & 12,137 & 2,000 \\
\hline & $\mathrm{CE}$ & 28730.292 & 4 & 7182573 & 32,842 & 2000 \\
\hline \multirow{2}{*}{$\begin{array}{l}\text { Engenharia } \\
\text { Grupo IV }\end{array}$} & FG & 5169673 & 4 & 1292418 & 3.859 & 2,004 \\
\hline & $\mathrm{CE}$ & 13209,742 & 4 & 3302,435 & 12,227 & 0,000 \\
\hline \multirow{2}{*}{$\begin{array}{l}\text { Engenharia } \\
\text { Grupo V }\end{array}$} & FG & 4562489 & 4 & 1140622 & 4650 & 0,001 \\
\hline & $\mathrm{CE}$ & 7297064 & 4 & 1824266 & 7,931 & 0,000 \\
\hline \multirow{2}{*}{$\begin{array}{l}\text { Engenharia. } \\
\text { Grupo VI }\end{array}$} & FG & 29060,144 & 4 & 7265,036 & 27,360 & 0,000 \\
\hline & $\mathrm{CE}$ & 81481675 & 4 & 20370,419 & 94631 & 2,000 \\
\hline \multirow{2}{*}{$\begin{array}{l}\text { Engenharia } \\
\text { Grupo VII }\end{array}$} & FG & 5977.761 & 4 & 1494440 & 4,982 & 0,001 \\
\hline & $\mathrm{CE}$ & 6205.134 & 4 & 1551283 & 8,170 & 0,000 \\
\hline \multirow{2}{*}{$\begin{array}{l}\text { Engenharia } \\
\text { Grupo VIII }\end{array}$} & FG & 1181.475 & 4 & 295,369 & 610 & 0,655 \\
\hline & $\mathrm{CE}$ & 2576839 & 4 & 644,210 & 2,740 & 0,027 \\
\hline
\end{tabular}

notas no Componente Específico se referem aos conteúdos específicos de cada área. Assim, Tabela 7 apresenta as estatísticas referentes à análise multivariada com as variáveis anteriormente relatadas. 
Tabela 7. Análise de variância fatorial múltipla para testar diferenças de desempenho entre os estudantes segundo o gênero

\begin{tabular}{|c|c|c|c|c|c|c|}
\hline $\begin{array}{l}\text { Fonte de } \\
\text { variação }\end{array}$ & $\begin{array}{c}\text { Nota nas } \\
\text { partes da prova }\end{array}$ & $\begin{array}{c}\text { Soma de } \\
\text { quadrados }\end{array}$ & $\begin{array}{l}\text { Graus de } \\
\text { liberdade }\end{array}$ & Quadrado Médio & Estatística $F$ & $\begin{array}{l}\text { Significância } \\
\text { do teste }(p)\end{array}$ \\
\hline \multirow{2}{*}{ Intercepto } & FG & 127346651,190 & 1 & 127346651,190 & 362967,947 & $0_{2}, 000$ \\
\hline & $\mathrm{CE}$ & 35474999,352 & 1 & 35474999,352 & 143845.850 & 0,000 \\
\hline \multirow{2}{*}{$\begin{array}{l}\text { Gênero e } \\
\text { Etnia }\end{array}$} & FG & 3402,092 & 1 & 3402,092 & 9,697 & 0,002 \\
\hline & $\mathrm{CE}$ & 858,833 & 1 & 858,833 & 3,482 & 0,062 \\
\hline \multirow{2}{*}{$\begin{array}{l}\text { Gênero e } \\
\text { renda }\end{array}$} & FG & 27195,420 & 2 & 13597,710 & 38,757 & 0,000 \\
\hline & $\mathrm{CE}$ & 22477,347 & 2 & 11238,674 & 45,571 & 0.000 \\
\hline \multirow{2}{*}{$\begin{array}{l}\text { Etnia e } \\
\text { renda }\end{array}$} & FG & 9895,748 & 2 & 4947,874 & 14,103 & 0.000 \\
\hline & $\mathrm{CE}$ & 23885,721 & 2 & 11942,860 & 48,427 & $0_{2} 000$ \\
\hline \multirow{2}{*}{$\begin{array}{l}\text { Gênero, } \\
\text { Etnia e } \\
\text { renda }\end{array}$} & FG & 878.070 & 2 & 439,035 & 1.251 & 0.286 \\
\hline & $\mathrm{CE}$ & 311,033 & 2 & 155,517 & 0.631 & 0.532 \\
\hline \multirow{2}{*}{ Erro } & FG & 72222803,858 & 205852 & 350,848 & & \\
\hline & $\mathrm{CE}$ & 50766842,153 & 205852 & 246,618 & & \\
\hline \multirow{2}{*}{ Total } & FG & 693098551,380 & 205865 & & & \\
\hline & $\mathrm{CE}$ & 261120178,200 & 205865 & & & \\
\hline
\end{tabular}

Verifica-se que há efeito significativo devido à interação entre o momento do curso, o gênero e a etnia apenas na parte de formação geral da prova. Além disso, existe uma interação altamente significativa, nas duas partes da prova, entre gênero e faixa de renda salarial, assim como para etnia e faixa de renda salarial. Esses resultados indicam que parte da variância do desempenho está sistematicamente associada às características dessas variáveis. Porém, quando verificada a contribuição de todas as variáveis, concomitantemente, nota-se que não há efeito significativo para o desempenho, em nenhuma das partes da prova.

Os resultados referentes à contribuição do gênero, etnia e nível socioeconômico revelam-se bastante relevantes na compreensão de outras variáveis associadas ao desempenho acadêmico. Além disso, assemelham-se a variáveis apontadas na literatura como de grande importância na investigação do sucesso ou fracasso acadêmico (RODRIGUES; BARBOSA; BLOIS, 2006; MARTÍN; DEL PRETTE, 2005; ALVES; BELTRÃO, 2004; ANDRADE; FRANCO; PITOMBEIRA, 2003; PRIMI; SANTOS; VENDRAMINI, 2002; CARVALHO, 2000; ALVES, 1998). Igualmente, esses estudos apontam a necessidade essencial de investigação de aspectos acadêmicos focados no estudante (como cognitivos, de aprendizagem, persistência, tempo de dedicação aos estudos), além de outros relacionados aos conteúdos ensinados, ação do professor em sala de aula, papel da família, entre outros. 


\section{CONCLUSÕES}

O estudo das variáveis relacionadas às diferenças de gênero em estudantes do ensino superior representa uma importante contribuição para a compreensão das variáveis associadas ao envolvimento acadêmico do universitário e ao desempenho bem sucedido. E esta importância é mais bem entendida ao pensar que o ensino superior pode se constituir em um importante acontecimento de vida, pelos desafios que coloca ao jovem estudante. Portanto, junto às exigências de sucesso acadêmico, necessárias para prosseguir nos estudos, o jovem precisa resolver questões relacionadas à consolidação da sua identidade, exploração e preparo para o investimento num projeto profissional.

A literatura em geral aponta que, para se ter uma maior compreensão a respeito das diferenças entre homens e mulheres neste campo, é preciso que sejam incluídas nos estudos algumas outras variáveis que têm mostrado uma influência significativa nos resultados dos testes educacionais, tais como: variáveis cognitivas e psicológicas; empenho nas tarefas acadêmicas; currículo e papel do professor; nível sócio-econômico; idade; e grau de escolaridade dos pais.

Portanto, o presente estudo não teve a pretensão de estabelecer uma relação causal entre o gênero e o desempenho acadêmico, mesmo porque se trata de uma avaliação educacional em larga escala que não possui elementos qualitativos suficientes que permitissem tamanha diferenciação. Além disso, como apontado nos demais estudos citados ao longo do texto, também se acredita que outras variáveis estão intrinsecamente relacionadas ao gênero, em um panorama socioeconômico mais amplo, como a etnia e o nível de renda familiar e que, desta forma, deveriam ser mais profundamente estudadas.

Como apontado nos resultados deste estudo, observou-se certa tendência de modificação ou até inversão de papéis em áreas reconhecidas tradicionalmente como estereotipadas. Isso indica que, muito provavelmente, o aumento da inserção de mulheres no Ensino Superior esteja refletindo não só na mudança de papéis e estereótipos, mas também no processo de ensino e nas relações sociais estabelecidas entre colegas e professores. Na medida em que há um espaço coletivo para reflexão de tendências e papéis acredita-se que se alteram também os modelos de avaliação, muitas vezes baseados nestas diferenças, na chamada bipolarização dos conhecimentos.

\section{REFERÊNCIAS}

ALMEIDA, L. S. et al. Acesso e sucesso no ensino superior em Portugal: Questões de género, origem sócio-cultural e percurso académico dos alunos. 
Psicologia: Reflexão e Crítica, Porto Alegre, v. 19, n. 3, p. 507-514, 2006. Disponível em: <www.scielo.br>. Acesso em: 30 ago. 2009.

ALVES, I. C. B. Variáveis significativas na avaliação da inteligência. Psicologia Escolar e Educacional, Campinas, v. 2, n. 2, p. 109-114, 1998.

ALVES, J. E. D.; BELTRÃO, K. I. A reversão do hiato de gênero na educação brasileira no século XX. In: ENCONTRO NACIONAL DE ESTUDOS POPULACIONAIS - ABEP, 14., Caxambu, 2004.

ANDRADE, D. F.; TAVARES, H. R.; VALLE, R. C. Teoria da resposta ao item: conceitos e aplicações. São Paulo: Associação Brasileira de Estatística, 2000 .

ANDRADE, M. S.; FRANCO, C.; PITOMBEIRA, J. B. Gênero e desempenho em matemática ao final do ensino médio: quais as relações. Estudos em Avaliação Educacional, São Paulo, v. 27, 2003. Disponível em: < http://www.fcc.org.br/pesquisa/publicacoes/ eae/arquivos/1110/ arquivoAnexado.pdf>. Acesso em: 15 abr. 2009

BARROSO, C. Metas de desenvolvimento do milênio, educação e igualdade de gênero. Cadernos de Pesquisa, São Paulo, v. 34, n. 123, 2004. Disponível em: $<$ http://www.fcc.org.br/pesquisa/publicacoes/ eae/ arquivos/1110/arquivoAnexado.pdf.> Acessado em: 15 abr. 2009.

BRANDÃO, Z. Fluxos escolares e efeitos agregados pelas escolas. Em Aberto, Brasília, v. 17, n. 71, p. 41-48, 2000.

BRASIL.MINISTÉRIO DA EDUCAÇÃO E CULTURA. Relatório

Técnico. Instituto Nacional de Estudos e pesquisas Educacionais Anísio Teixeira (INEP), 2005. Disponível em: <http://www.inep.gov.br/download/ enade/2005/relatorios/>. Acesso em: 15 abr. 2009.

CARVALHO, M. E. P. Relações entre família e escola e suas implicações de gênero. Cadernos de Pesquisa, São Paulo, v. 110, p. 143-155, 2000.

IBGE - Instituto Brasieliro de Geografia e Estatisticca. Censo do Instituto Brasileiro de Geografia e Estatística, 2000 Disponível em: < http://www. ibge.gov.br/>. Acesso em: 15 abr. 2009.

CÉSAR, C. C.; SOARES, J. F. Desigualdades acadêmicas induzidas pelo contexto escolar. Revista Brasileira de Estudos da População, São Paulo, v. 18 , p. $97-110,2001$. 
FERRÃO, M. E. F.; FERNANDES, C. O efeito-escola e a mudança - Dá para mudar? Evidências da investigação brasileira. Revista Electrónica sobre Calidad, Eficácia Y Cambio em Educación (REICE), v. 1, n. 1, 2003 [on line]. Disponível em: <http://www.ice.deusto.es/rinace/reice/vol1n1/ FerraoyFernandes.pdf $>$. Acesso em: 15 abr. 2009.

FORMIGA, N. S. O tipo de orientação cultural e sua influência sobre os indicadores do rendimento escolar. Psicologia: Teoria e Prática, São Paulo, v. 6, n. 1, p. 13-29, 2004.

MARTINI, M. L.; DEL PRETTE, Z. A. P. Atribuições de causalidade e afetividade de alunos de alto e baixo desempenho acadêmico em situações de sucesso e de fracasso escolar. Revista Interamericana de Psicologia, Porto Alegre, v. 39, n. 3, p. 355-368, 2005.

PRIMI, R.; SANTOS, A. A. A.; VENDRAMINI, C. M. M. Habilidades básicas e desempenho acadêmico em ingressantes universitários. Estudos de Psicologia, Natal, v. 17, n. 1, p. 47-55, 2002.

RIBEIRO, I.; ALMEIDA, L.; GOMES, C. Conhecimentos prévios, sucesso escolar e trajectórias de aprendizagem: do $1^{\circ}$ para o $2^{\circ}$ ciclo do ensino básico. Avaliação Psicológica, Porto Alegre, v. 2, p. 127-133, 2006.

RODRIGUES, F. P. M.; BARBOSA, C. I. V.; BLOIS, M. D. O processo de avaliação institucional como multiplicador de iniciativas para o aperfeiçoamento docente $-1^{\mathrm{a}}$ parte. Avaliação, Campinas, v. 11, n. 2, p. 151-166, 2006.

SAAVEDRA, L. Sucesso/ insucesso escolar: a importância do nível socioecnómico e do gênero. Psicologia, Minho, v. 15, n. 1, p. 67-92, 2001.

SOARES, J. F. O efeito da escola no desempenho cognitivo de seus alunos. Revista Electrónica Iberoamericana sobre Calidad, Eficacia y Cambio en Educación (REICE), v. 2, n. 2, 2004. Disponível em:<http://www.ice. deusto.es/rinace/reice/vol2n2/Soares.pdf>. Acesso em: 20 maio 2008.

URBINA, S. Essentials of psychological testing. New Jersey: John Wiley, 2004.

VIANNA, H. M. Avaliações nacionais em larga escala: análises e propostas. Estudos em Avaliação Educacional, São Paulo, v. 27, p. 41-76, 2003. 